$17^{\circ}$ ERGODESIGN \& USIHC 2019

PUC-Rio, 11 a 13 de dezembro

Rio de Janeiro, RJ, Brasil $17^{\circ}$ Ergodesign - Congresso Internacional de Ergonomia e Usabilidade de Interfaces Humano Tecnológica: Produto, Informações Ambientes Construídos e Transporte

$17^{\circ}$ USIHC - Congresso Internacional de Ergonomia e Usabilidade

de Interfaces Humano Computador

\title{
Um Estudo da Usabilidade da Ferramenta de Correio Eletrônico da Google
}

\author{
A Study of the Usability of Google's Email Tool \\ SANTA ROSA, José Guilherme \\ UFRN, Professor Associado \\ jguilhermesantarosa@gmail.com
}

CAVALCANTE GOMES DE OLIVEIRA, Deivson

UFPE, Mestrando em Ergonomia

deivsoncgo@hotmail.com

ARAÚJO WANDERLEY, Flávio

UFPE, Mestrando em Ergonomia

flaviowand@hotmail.com

SANTOS BATISTA, Carlos Eduardo

UFPE, Mestrando em Ergonomia

kaemirk@outlook.com

\section{RESUMO:}

O presente trabalho tem como objetivo avaliar a usabilidade da ferramenta Gmail, por meio de um estudo de natureza quali-quantitativa que considerou a realização de tarefas específicas. Para tanto, foram recrutados 20 participantes com níveis diferentes de experiência com a ferramenta. Foi solicitado aos participantes que respondessem um questionário contendo informações sóciodemográficas e de experiência com o uso de email. A análise das tarefas e da interação com o sistema foi realizada por meio de Teste de Usabilidade (10 usuários) e Avaliação Cooperativa (10 usuários). Foram avaliadas as tarefas: 1- criar uma conta de e-mail; 2- configurar uma assinatura de rodapé; 3- criar um marcador (pasta) e organizar e-mails recebidos na pasta recém criada; 4- criar uma agenda e enviar convite de um evento para participantes; 5 - configurar o tempo de cancelamento de e-mails enviados para 20 segundos. Após a realização de cada teste de usabilidade foi aplicado um questionário adaptado do Questionnarie Interaction Satisfaction (Shneiderman, 2005) a respeito da percepção de cada participante sobre a interação com o Gmail. Verificou-se correlação entre problemas de usabilidade, duração da tarefa e a percepção dos usuários a respeito da satisfação durante a interação com o sistema.

Usabilidade, Avaliação Cooperativa, Correio Eletrônico. 
$17^{\circ}$ ERGODESIGN \& USIHC 2019

PUC-Rio, 11 a 13 de dezembro Rio de Janeiro, RJ, Brasil $17^{\circ}$ Ergodesign - Congresso Internacional de Ergonomia e Usabilidade de Interfaces Humano Tecnológica: Produto, Informações Ambientes Construídos e Transporte

$17^{\circ}$ USIHC - Congresso Internacional de Ergonomia e Usabilidade

de Interfaces Humano Computador

\begin{abstract}
This paper has a qualitative-quantitative nature with the aim of evaluating the usability of the Gmail tool during the execution of certain tasks. To this end, 20 participants were recruited with different levels of expertise about the resources offered by the tool. The participants were asked to respond a questionnaire containing socio-demographic information and experiences in using email. The analysis of the tasks and the interactions with the system was performed through the Usability Test (10 users) and Cooperative Assessment (10 users). The following tasks were analyzed: 1-creating an e-mail account; 2- setting up a footnote signature; 3- creating a bookmark (folder) and organizing incoming emails in the newly created folder; 4- creating a calendar and sending an invitation to an event to the other participants; 5- setting up cancel time for sent emails to 20 seconds. After each usability test, an adapted questionnaire from Questionnaire Interaction Satisfaction (Shneiderman, 2005) was applied to each participant, with the aim of capturing their perceptions regarding the interaction with Gmail. We found a correlation between usability problems, task duration and users' satisfaction perception during the interaction with the system.
\end{abstract}

Usability, Cooperative Evaluation, Mail Tool.

\title{
1. INTRODUÇÃO
}

A mensagem eletrônica ou e-mail foi uma das aplicações que caracterizaram o primeiro período das redes de computadores, tendo sido introduzida em 1972. Uma aplicação tão importante que continua a existir até hoje, nos tempos das redes sociais, assumindo diversas formas. Seu formato básico continua preservado, com o endereço eletrônico do destinatário e a mensagem correspondente. Alguns recursos adicionais, como a anexação de arquivos e a formatação do texto (fontes, cores, destaques, ícones, emoticons) foram adicionados com o decorrer dos anos. Mas o conceito se perpetua de modo surpreendente após várias décadas (LINS,2013). Atualmente, o serviço de correio eletrônico é uma ferramenta acessível e onipresente de comunicação pela Internet. Esta ferramenta representa um recurso que oferece aos seus usuários, além de troca de mensagens e envio de documentos, a segurança e formalização do registro das mensagens enviadas, compartilhamento de arquivos e informação em larga escala, comunicação multimídia, dentre outros. Além disso, o usuário frequentemente utiliza o perfil de sua conta de email para acessar redes sociais e sites de comércio eletrônico pela web (SCHEN e col., 2013).

\section{$1.1 \quad$ O Gmail}




\section{$17^{\circ}$ ERGODESIGN \& USIHC 2019}

PUC-Rio, 11 a 13 de dezembro

Rio de Janeiro, RJ, Brasil $17^{\circ}$ Ergodesign - Congresso Internacional de Ergonomia e Usabilidade de Interfaces Humano Tecnológica: Produto, Informações Ambientes Construídos e Transporte

$17^{\circ}$ USIHC - Congresso Internacional de Ergonomia e Usabilidade

de Interfaces Humano Computador

A ferramenta Gmail, desenvolvida pelo Google, é atualmente a principal ferramenta de correio eletrônico para web. Seu acesso é público e gratuito e tem sido utilizado por mais de 1,5 bilhão de usuários em todo o mundo. Segundo Calore (2013), o Gmail tornou-se popular por apresentar ao usuário uma interface mais engenhosa, adicionando recursos por meio de extensões similares a aplicativos. Neste sentido, usabilidade das interfaces dos serviços de email tem sido objeto de atenção considerando a grande utilização desta ferramenta no cotidiano profissional e social de seus usuários (WENTZ e col., 2013).

Figura 1- Menu lateral e área de texto do Gmail

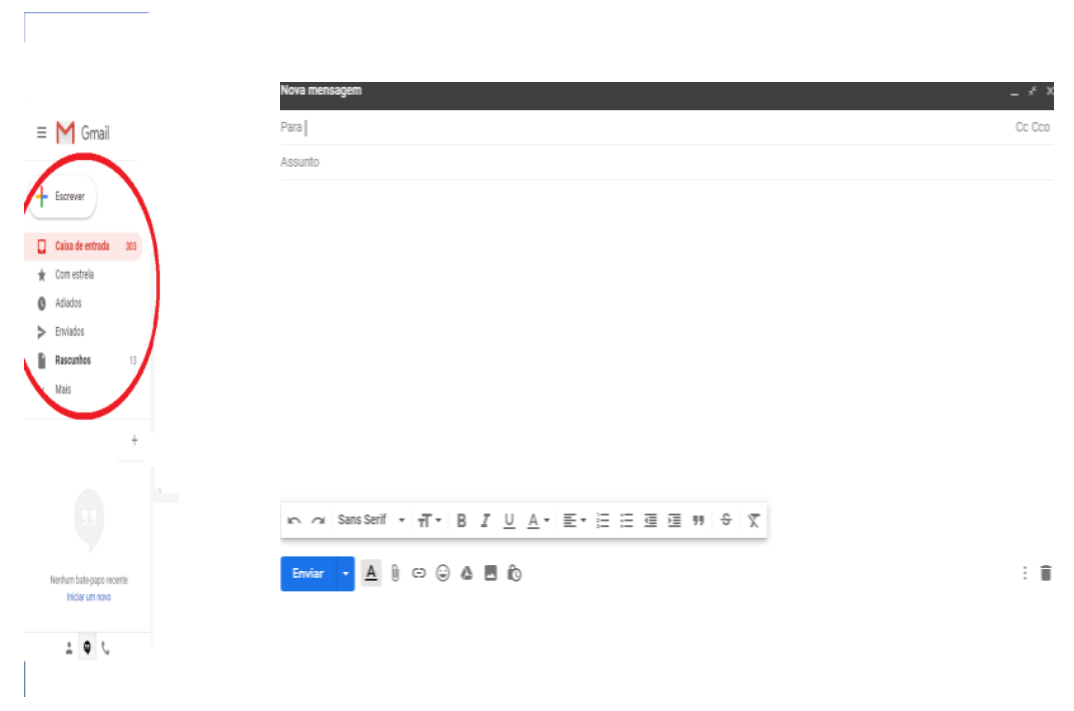

A Figura 1 exibe o menu lateral e a área de texto do Gmail com algumas funções tais como:

- Caixa de entrada: visualizar e-mails;

- Com estrela: Marcar e-mails importantes;

- Adiados: Consulta e cancela o adiamento de qualquer mensagem;

- Enviados:Consulta e-mails enviados pelo usuário;

- Rascunhos: Armazena e-mails em preparação.

\subsection{Usabilidade}




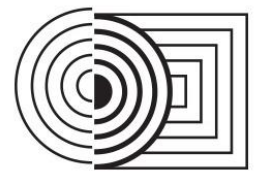

$17^{\circ}$ ERGODESIGN \& USIHC 2019

PUC-Rio, 11 a 13 de dezembro Rio de Janeiro, RJ, Brasil $17^{\circ}$ Ergodesign - Congresso Internacional de Ergonomia e Usabilidade de Interfaces Humano Tecnológica: Produto, Informações Ambientes Construídos e Transporte

$17^{\circ}$ USIHC - Congresso Internacional de Ergonomia e Usabilidade

de Interfaces Humano Computador

A NBR ISO 9126 define usabilidade como a capacidade de um software ser entendido, aprendido, utilizado e atrativo para o usuário, sendo usado sob condições específicas. A usabilidade é uma qualidade de uso, ela pode ser definida ou medida para um contexto específico no qual um sistema é utilizado. Um usuário experiente poderá ter uma boa usabilidade enquanto um iniciante poderá ter uma péssima experiência, ou vice-versa; ou ainda, o sistema pode ser fácil de usar se o sistema for usado eventualmente, mas difícil se for utilizado continuamente. (CYBIS, 2003).

Para Nielsen (1994), o sistema para ter uma boa usabilidade é preciso que atenda aos seguintes requisitos: ser de fácil aprendizagem, ser eficiente na utilização, ser fácil de lembrar, ter poucos erros e satisfazer subjetivamente. Os cinco critérios compõem a natureza multidimensional da usabilidade.

Segundo Cybis (2003), o desenvolvimento de sistemas com boa usabilidade pode gerar um impacto positivo na tarefa, no sentido da eficiência, eficácia e produtividade, permitindo ao usuário atingir seus objetivos com menos esforço e mais satisfação. Uma interface difícil de ser utilizada eleva a carga cognitiva do usuário trazendo consequências negativas que vão desde a resistência ao uso, até a subutilização, e chega ao ponto do usuário abandonar o sistema. A depender da dimensão em que o software é empregado os prejuízos podem ser elevados. Santa Rosa, Pereira Junior e Lameira (2016) ressaltam que para projetar a experiência do usuário de um sistema, deve-se, considerar além dos objetivos pragmáticos do usuário, as emoções desencadeadas durante a interação com elementos de interface. Estes aspectos podem ser determinantes do engajamento com o sistema mas, também, da desistência do usuário em utilizá-lo.

Sendo assim, considerando a relevância do uso do correio eletrônico na comunidade em geral bem como a ampla utilização do serviço de e-mail desenvolvido pela Google, este artigo tem como objetivo avaliar a usabilidade da ferramenta Gmail durante a realização de determinadas tarefas relacionadas com a criação de conta de email, organização de mensagens recebidas em marcadores (pastas), agendamento de eventos, e configuração de cancelamento de email enviado, por meio de um estudo que considerou tanto aspectos quantitativos e métricas de usabilidade quanto da satisfação subjetiva do usuário.

\section{METODOLOGIA}

Trata-se do relato de um estudo de natureza quali-quantitativa. A amostra foi composta por vinte participantes, recrutados de forma aleatória, que apresentam diferentes níveis de experiência com os recursos oferecidos pela ferramenta Gmail.

O estudo foi realizado numa sala com acesso à internet, desktop, com a participação do moderador e o pesquisado. Por não ter acesso a uma sala específica para a realização do estudo foi necessário que o moderador utilisasse notebook para visualizar a tela do sistema e facilitar a interação com o participante. Não foi considerado utilizar celular pela dificuldade de visualização da tela pelo moderador o que poderia prejudicar a avaliação da usabilidade do correio eletrônico. 
Escolheu-se trabalhar com usuários e não usuários do correio eletrônico Gmail para mostrar a percepção do usuário a respeito da facilidade do uso do sistema. Mesmo que o participante não tenha tido contato com sistema, seu uso com determinada frequência, deixa o usuário mais acostumado com os problemas de usabilidade e até deixe de percebê-los. Enquanto opera o usuário vai aprendendo a lidar com sistema e consegue utilizá-lo, mas quando o mesmo não tem o conhecimento da ferramanta pode-se constatar se o sistema é de fato intuitivo.Inicialmente, foi solicitado aos participantes o preenchimento de um questionário de background contendo informações sócio-demográficas e de experiência com a uso de ferramentas do correio eletrônico do Gmail e/ou outros.

A análise das tarefas e da interação com o sistema foi realizada por meio da aplicação das técnicas: a) Teste de Usabilidade (RUBIN e CHISNELL, 2008) com 10 usuários participantes e b) Avaliação Cooperativa (MONK e col., 1993) com 10 usuários participantes. Para realizar o Teste de Usabilidade, os usuários participantes utilizaram o Gmail por meio de um navegador web instalado em microcomputador com acesso à Internet. A condução dos testes de usabilidade e da Avaliação Cooperativa contou com a participação de um colaborador e, também de um moderador do teste, que ficou responsável por enviar e-mails aos participantes em tempo real durante a interação com a ferramenta. A figura 2 exibe a interface do Gmail utilizada pelos pesquisados.

Figura 2- Interface do Gmail

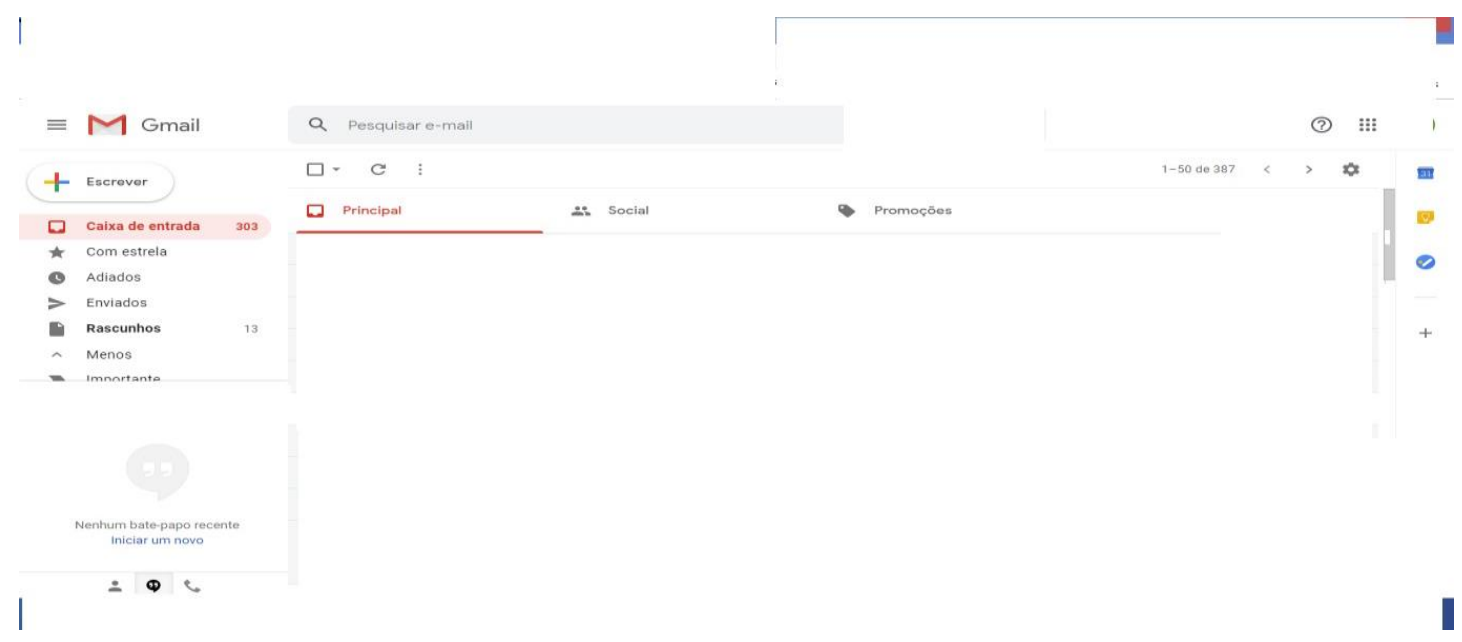

As tarefas, tanto para a realização das sessões de Teste de Usabilidade, quanto para a Avaliação Cooperativa, foram definidas a partir da identificação das principais atividades disponíveis aos usuários da ferramenta, e são descritas a seguir: 1- criar uma conta de email; 2- configurar uma assinatura de rodapé; 3- criar um marcador (pasta) e organizar emails recebidos na pasta recém criada; 4- criar uma agenda e enviar convite de um evento para três participantes; 5 - configurar o tempo de cancelamento de e-mails enviados para vinte segundos. Após a realização de cada de Teste de Usabilidade foi aplicado um Questionário de Satisfação, adaptado do Questionnarie Interaction Satisfaction (Shneiderman, 2005) a respeito da percepção de cada participante sobre a interação com o 
$17^{\circ}$ ERGODESIGN \& USIHC 2019

PUC-Rio, 11 a 13 de dezembro Rio de Janeiro, RJ, Brasil $17^{\circ}$ Ergodesign - Congresso Internacional de Ergonomia e Usabilidade de Interfaces Humano Tecnológica: Produto, Informações Ambientes Construídos e Transporte

$17^{\circ}$ USIHC - Congresso Internacional de Ergonomia e Usabilidade de Interfaces Humano Computador

Gmail. A figura 3 mostra a interface utilizada pelos usuários para a realização de tarefas como a criação de conta, assinatura de rodapé e a criação de agenda.

Figura 3- Interface para a realização das tarefas
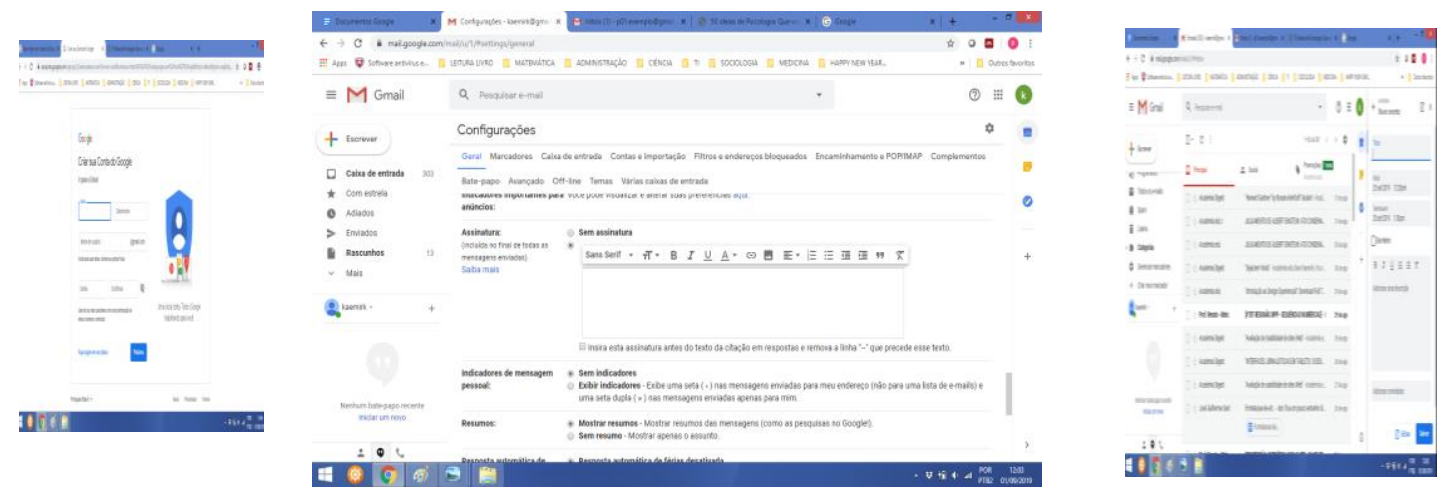

\section{RESULTADOS E DISCUSSÃO}

A amostra deste estudo foi composta por participantes de ambos os sexos (13 do sexo masculino e 07 do sexo feminino) com idades variando de 15 a 62 anos. Quanto à escolaridade, 10 participantes possuíam pós-graduação, 05 ensino superior e 05 ensino médio.

\section{Gráfico 1- Frequência de uso da Internet}

Frequência de uso da internet

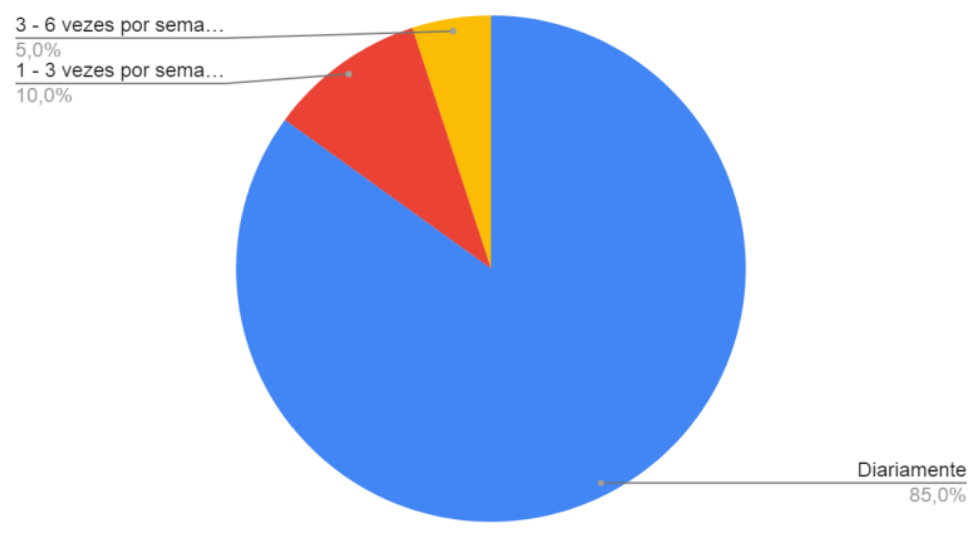




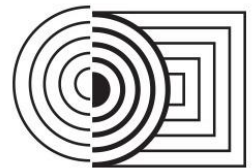

\section{$17^{\circ}$ ERGODESIGN} \& USIHC 2019

PUC-Rio, 11 a 13 de dezembro Rio de Janeiro, RJ, Brasil $17^{\circ}$ Ergodesign - Congresso Internacional de Ergonomia e Usabilidade de Interfaces Humano Tecnológica: Produto, Informações Ambientes Construídos e Transporte

$17^{\circ}$ USIHC - Congresso Internacional de Ergonomia e Usabilidade de Interfaces Humano Computador

Com relação ao nível de experiência com o uso da Internet, a grande maioria (85\%) dos usuários participantes deste estudo acessam a Internet diariamente. Contudo, $35 \%$ relatam utilizar os serviços de email diariamente, $40 \%$ de 1 a 3 vezes na semana e $25 \%$ de 3 a 6 vezes (Gráfico 1).

O Serviço de e-mail da Google foi apontado como o segundo mais utilizado de forma prioritária (40\% dos usuários), ao passo que o Hotmail/Outlook foi o provedor mais escolhido para uso prioritário pela população estudada (50\% dos participantes).

Quando perguntados sobre a utilização de um segundo email, 54\% dos participantes relatam usar o serviço do Gmail para essa finalidade. Ao analisar o nível de experiência dos participantes com os recursos oferecidos pelo Gmail escolhidos como objeto deste estudo, observou-se que $30 \%$ dos participantes não utilizam estas funcionalidades. A atividade mais utilizada foi a organização de emails recebidos na caixa de entrada em pastas, seguida por assinatura, criação de agenda de eventos, e cancelamento de email enviado.

Quando questionados sobre a finalidade da utilização dos serviços de correio eletrônico, grande parte dos respondentes indicaram a troca de mensagens e arquivos pessoais $(85 \%)$ e do trabalho $(80 \%)$ como principal propósito, seguido de compras de produtos e serviços online $(60 \%)$, e acesso a redes sociais (35\%). Os principais motivos da escolha pelo email entre as diversas ferramentas de comunicação online apontados pelos usuários desta amostra foram a disponibilidade de acesso por diferentes dispositivos, tais como desktop, tablets e smartphones (85\%) e a facilidade do uso (75\%).

A primeira tarefa analisada consistiu em criar uma conta no Gmail sendo solicitado ao usuário a criação de um determinado email e senha. Os usuários não apresentaram nenhuma dificuldade em encontrar o site do Gmail e iniciar a realização da tarefa. Todos os participantes conseguiram concluir a tarefa, entretanto após um erro de digitação da idade na ocasião em que um dos usuários cadastrou uma data de nascimento equivocada, como pessoa menor de idade, o sistema de autenticação do Gmail não permitiu a conclusão do cadastro sem que o usuário incluísse um email de um responsável, mesmo após a devida correção.

Para concluir a tarefa, foi necessário que o participante acessasse o Gmail por outro microcomputador, provavelmente por motivos de registro de IP da máquina utilizada anteriormente mesmo após reiniciá-la. Os participantes que realizaram Teste de Usabilidade conseguiram concluir esta tarefa sem maiores dificuldades, em média, após 06 minutos, mesmo tempo aproximado para os usuários que realizaram a avaliação cooperativa. Dentre os problemas apresentados, observamos que apenas quatro usuários (dois do grupo avaliado por meio do teste de usabilidade e dois de avaliação cooperativa) utilizaram o recurso de visualizar senha. Um dos usuários, que foi avaliado por meio da avaliação cooperativa, não utilizou este recurso mesmo após errar a confirmação da senha por diversas vezes durante o preenchimento do formulário.

Quando questionado se o ícone desta funcionalidade apresentado na tela do Gmail poderia indicar um recurso que facilitasse a compreensão do erro o mesmo relatou não ter feito essa associação claramente. Entretanto, alguns usuários relataram ter conhecimento deste recurso embora não tenha o hábito de utilizá-lo.

Observou-se também que após a finalização da tarefa, o Gmail apresentou opções de exibição de caixa de entrada no idioma inglês de acordo com a figura 4, causando alguma dificuldade de compreensão em boa parte dos usuários, muitos deles não compreenderam exatamente a diferença entre elas. A mudança de idioma representou uma limitação 


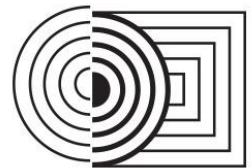

$17^{\circ}$ ERGODESIGN \& USIHC 2019

PUC-Rio, 11 a 13 de dezembro Rio de Janeiro, RJ, Brasil $17^{\circ}$ Ergodesign - Congresso Internacional de Ergonomia e Usabilidade de Interfaces Humano Tecnológica: Produto, Informações Ambientes Construídos e Transporte

$17^{\circ}$ USIHC - Congresso Internacional de Ergonomia e Usabilidade de Interfaces Humano Computador

importante especialmente porque todo o cadastro anterior já foirealizado em português, de forma que o usuário não compreendeu o motivo desta mudança.

Figura 4- Interface do Gmail ao criar a conta de e-mail

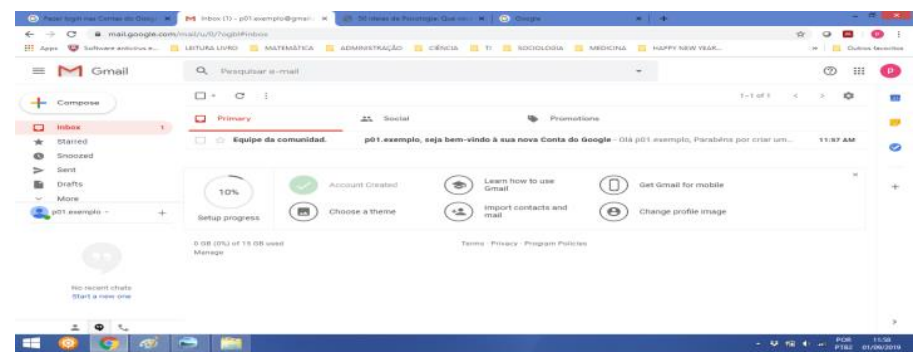

Para realizar a segunda tarefa, foi solicitado aos participantes que criassem uma assinatura para o email. De acordo com a descrição do Gmail, uma assinatura de e-mail é um texto, como suas informações de contato ou uma citação favorita, adicionado automaticamente ao final das mensagens do Gmail, como um rodapé. Além de texto, também é possível acrescentar imagens.

Dos 20 participantes deste estudo, apenas a metade conseguiu realizar a tarefa sem apresentar nenhuma dificuldade e os demais apresentaram algum grau de dificuldade para localizar rapidamente onde criar a assinatura. Entre os usuários que foram submetidos a avaliação cooperativa, dois tiveram a necessidade de intervenções dos moderadores para concluir a tarefa, e um usuário utilizou o recurso de busca na ajuda do Gmail. Em média, os participantes necessitaram de quatro minutos para identificar o local para editar a assinatura e realizar a atividade.

Observou-se que seis usuários procuraram alterar as configurações de assinatura no menu da caixa de entrada e nas pastas de emails, dispostas à esquerda da tela. Por meio da avaliação cooperativa, foi possível constatar que usuários imaginavam que as funções do e mail estavam dispostas no lado esquerdo da tela, juntamente com as pastas de email (caixa de entrada, emails enviados, lixeira, caixa de spam e marcadores), não percebendo de forma intuitiva que o menu de configurações está disposto à direita.

Quanto à tarefa de criar um marcador (pasta) e organizar emails recebidos no marcador recém criado, os resultados deste estudo indicam que o termo utilizado pelo Gmail parece não ser facilmente compreendido pelos participantes deste estudo como uma pasta, considerando que boa parte (73\%) dos pesquisados relataram dificuldade em compreender o termo, associando muitas vezes à sinalização de emails da caixa de entrada. Muitos usuários participantes deste estudo (60\%) apesar de concluírem a tarefa, relataram não localizar de forma clara a opção criar um novo marcador pois ao clicar em "Mais" nas opções de pastas à esquerda da tela as opções não surgem de forma intuitiva, sendo necessário perceber que existem novas opções por meio do surgimento de uma barra de rolagem (figura 5). Um dos participantes submetido ao teste de usabilidade desistiu do teste e não concluiu a tarefa por não encontrar esta opção na tela inicial do Gmail. 
$17^{\circ}$ ERGODESIGN \& USIHC 2019

PUC-Rio, 11 a 13 de dezembro Rio de Janeiro, RJ, Brasil $17^{\circ}$ Ergodesign - Congresso Internacional de Ergonomia e Usabilidade de Interfaces Humano Tecnológica: Produto, Informações Ambientes Construídos e Transporte

$17^{\circ}$ USIHC - Congresso Internacional de Ergonomia e Usabilidade de Interfaces Humano Computador

Figura 5- Problema usabilidade em localizar tarefa criar marcadores

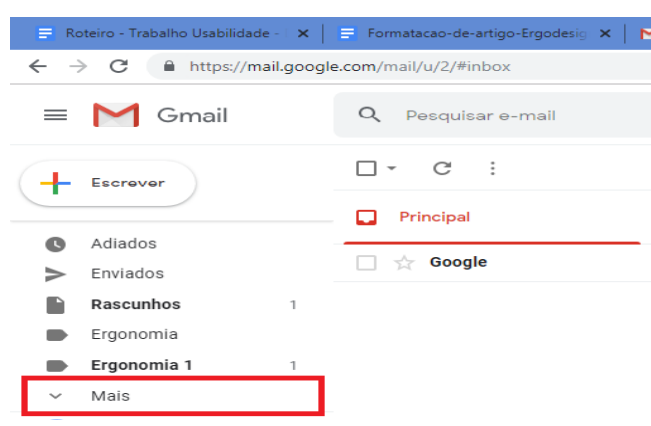

Observou-se também que para concluir a tarefa, dois usuários criaram novos marcadores utilizando o menu de configurações do Gmail. Para movimentar as mensagens recebidas para as pastas, parte dos usuários utilizaram do recurso "clicar e arrastar", outros sinalizaram a mensagem e clicaram em "mover para" e outros utilizaram diretamente a opção marcadores.Com relação a tarefa criar uma agenda de reunião em data e horário determinado e enviar convite a três participantes, todos os usuários conseguiram finalizar a ação, apesar de identificarem alguns problemas quanto a sua execução. Os usuários conseguiram executar a tarefa, em média, após 06 minutos, entretanto apenas 05 participantes não apresentaram nenhuma dificuldade. $O$ tamanho do ícone relativo à agenda, localizado no canto superior direito foi a queixa relatada mais frequente (06 participantes).

Com relação a tarefa criar uma agenda de reunião em data e horário determinado e enviar convite a 03 participantes, todos os usuários conseguiram finalizar a ação, apesar de identificarem alguns problemas quanto a sua execução. Os usuários conseguiram executar a tarefa, em média, após 06 minutos, entretanto apenas 05 participantes não apresentaram nenhuma dificuldade. $O$ tamanho do ícone relativo à agenda, localizado no canto superior direito (figura 6 ) foi a queixa relatada mais frequente (06 participantes). 
Figura 6 -ícone da agenda do Gmail

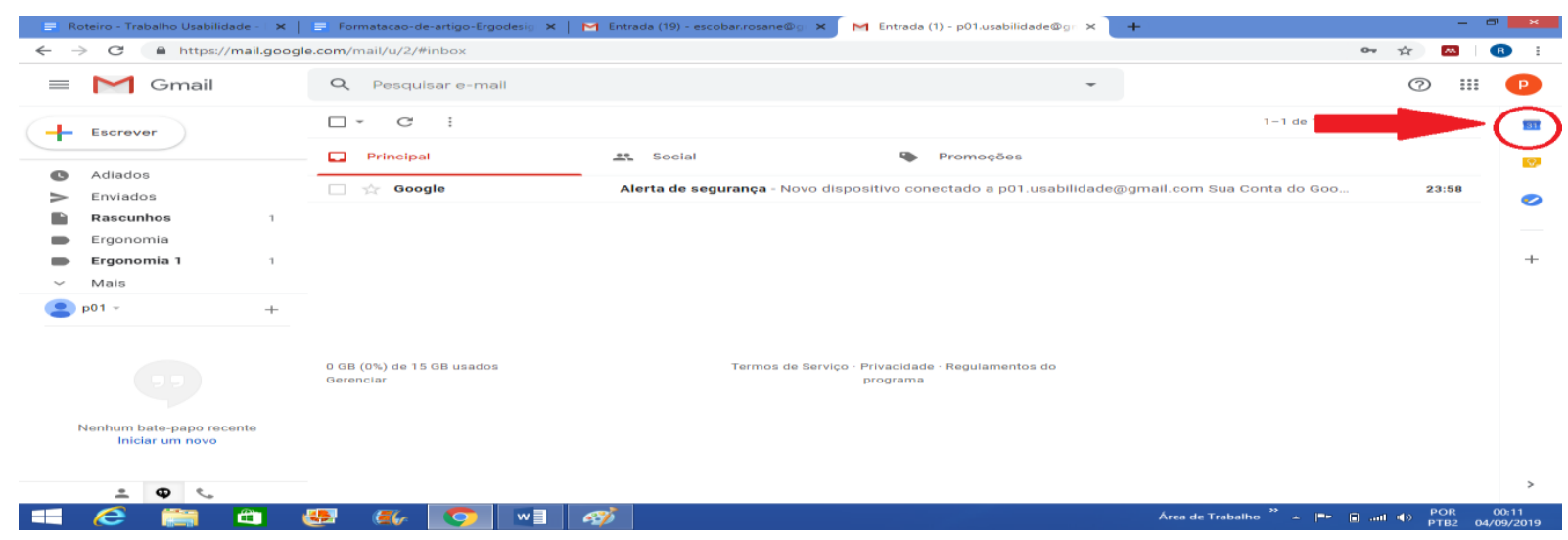

Quatro participantes apresentaram dificuldades para adicionar os contatos dos convidados das reuniões, tendo em vista que os contatos eram inseridos de forma diferente da forma usualmente utilizada para enviar email a vários contatos, por meio do uso do “;”. Alguns usuários enviaram a sequência de emails sem perceber que os emails estavam inválidos. Pois, o Gmail, apesar de sinalizar que o email não é válido, permite o envio dos convites (figura 7). Nessa situação apenas o último email da sequência separada por meio do uso do ponto e vírgula foi enviado corretamente para o convidado.

Figura 7 - Envio de convites para um evento

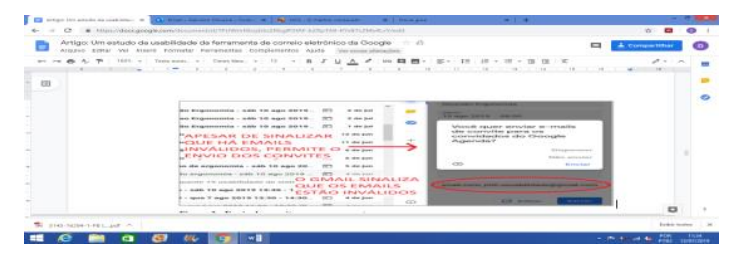

Além disso, um participante tentou localizar esta função digitando o termo agenda na caixa de busca, sem perceber que essa busca se referia a localizar determinado email entre os recebidos ou enviados e não com a busca pela ajuda do Gmail.

Na última tarefa avaliada, foi solicitado aos participantes alteração da definição de tempo de cancelamento de email enviado para 20 segundos. A maioria dos participantes (50\%) não apresentou nenhuma dificuldade para executar a tarefa, identificando rapidamente a função de alteração do período de cancelamento do email no menu configurações. Entretanto, 20\% dos usuários tentaram localizar a tarefa próximo as pastas de email, à esquerda da tela 
$17^{\circ}$ ERGODESIGN \& USIHC 2019

PUC-Rio, 11 a 13 de dezembro Rio de Janeiro, RJ, Brasil $17^{\circ}$ Ergodesign - Congresso Internacional de Ergonomia e Usabilidade de Interfaces Humano Tecnológica: Produto, Informações Ambientes Construídos e Transporte

$17^{\circ}$ USIHC - Congresso Internacional de Ergonomia e Usabilidade

de Interfaces Humano Computador

principal, assim como observado na tarefa criar assinatura de email. Apenas um usuário não conseguiu concluir a tarefa, após tentar localizar, sem sucesso, no menu de configurações e na caixa de entrada.

De acordo com o quadro 1, observa-se que a tarefa com maior tempo médio de execução pelos usuários que realizaram o teste de usabilidade é a de criar conta de e-mail com média de 05:57 minutos e desvio padrão de 03:57. Enquanto a tarefa com menor tempo médio de realização é a configuração do tempo de cancelamento com média de 01:34 minutos e desvio padrão de 01:05 minutos.

Quadro 1. Tempo médio e desvio padrão das cinco tarefas realizadas no teste de usabilidade

\begin{tabular}{|c|c|c|}
\hline TAREFA & TEMPO MÉDIO & DESVIO PADRÃO \\
\hline Criar conta de e-mail & $05: 57$ & $03: 57$ \\
\hline Assinatura de rodapé & $03: 14$ & $01: 30$ \\
\hline $\begin{array}{c}\text { Criar marcador e enviar e-mail para o } \\
\text { mesmo }\end{array}$ & $03: 08$ & $02: 20$ \\
\hline Criar agenda e enviar convite & $05: 39$ & $01: 05$ \\
\hline Configurar tempo de cancelamento & $01: 34$ & \\
\hline
\end{tabular}

\section{Fonte: autores}

Como pode ser observado no quadro 2 , a tarefa com maior tempo de execução é a criação de agenda e envio de convite com tempo médio de 06:46 minutos e desvio padrão de 02:21 minutos e a tarefa com o menor tempo de execução é configuração do tempo de cancelamento de envio de e-mail com média de 02:53 e desvio padrão de 01:34.

Quadro 2. Tempo médio e desvio padrão das cinco tarefas realizadas na avaliação cooperativa

\begin{tabular}{|c|c|c|}
\hline TAREFA & $\begin{array}{c}\text { TEMPO } \\
\text { MÉDIO }\end{array}$ & DESVIO PADRÃO \\
\hline Criar conta de e-mail & $05: 35$ & $02: 11$ \\
\hline Assinatura de rodapé & $05: 06$ & $01: 50$ \\
\hline $\begin{array}{c}\text { Criar marcador e enviar e-mail para o } \\
\text { mesmo }\end{array}$ & $03: 16$ & $02: 21$ \\
\hline Criar agenda e enviar convite & $06: 46$ & 030 \\
\hline
\end{tabular}




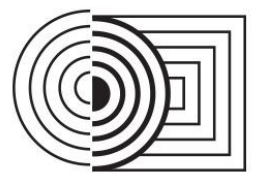

$17^{\circ}$ ERGODESIGN \& USIHC 2019

PUC-Rio, 11 a 13 de dezembro Rio de Janeiro, RJ, Brasil

$17^{\circ}$ Ergodesign - Congresso Internacional de Ergonomia e Usabilidade de Interfaces Humano Tecnológica: Produto, Informações Ambientes Construídos e Transporte

$17^{\circ}$ USIHC - Congresso Internacional de Ergonomia e Usabilidade de Interfaces Humano Computador

\begin{tabular}{|c|c|c|}
\hline Configurar tempo de cancelamento & $02: 53$ & $01: 34$ \\
\hline
\end{tabular}

Fonte: autores

Com a aplicação do Questionário de Satisfação, pode-se observar que, em uma escala likert de 10 pontos obteve-se média de 7,79 para a satisfação geral com o sistema Gmail. $\mathrm{Na}$ primeira parte do questionário, que envolveu três questões relativas à reação do sistema, a satisfação dos usuários com o Gmail teve média de 7,18. Na segunda parte, que avalia em cinco questões fatores relacionados à tela do sistema (forma e tamanho de letras,

organização, quantidade de informação, e sequência de apresentação das telas) a média da satisfação dos usuários foi de 6,99. A terceira parte do questionário avaliou a terminologia empregada e informações apresentadas pelo sistema com média de 7,63. Na quarta parte, os usuários responderam duas questões relativas ao aprendizado e utilização do sistema, que teve uma média de 5,16. Por fim, na última parte, os usuários responderam três questões relativas a Capacidades do Sistema (velocidade, correção de erros e confiabilidade com o sistema) com média de 7,74.

Gráfico 2- Grau de satisfação do usuário com o sistema

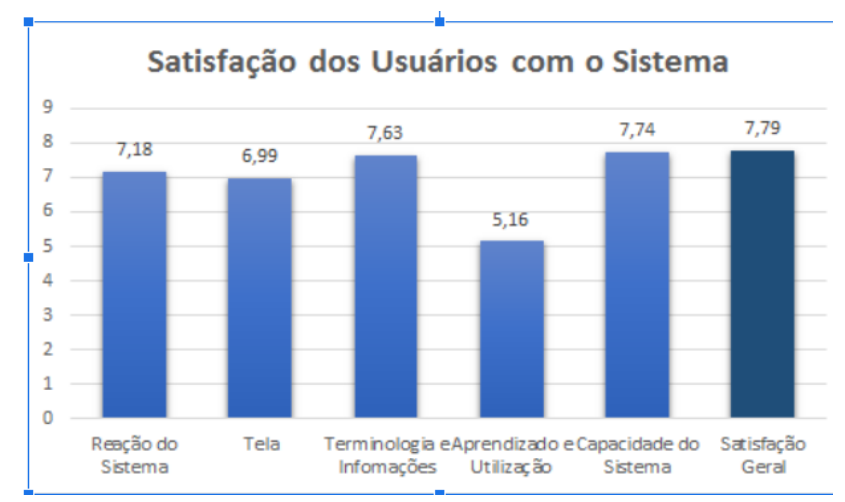

Desta forma, pode-se observar que os usuários apresentaram melhor grau de satisfação na interação com o sistema do Gmail em questões relacionadas à Capacidade do Sistema, seguida da Terminologia empregada e informações apresentadas pelo sistema, reação do sistema, tela e aprendizado e utilização do sistema.

O baixo nível de satisfação com os fatores relacionados ao aprendizado e utilização do sistema evidenciam a necessidade de promover melhorias na interface que tornem seus recursos e funcionalidades mais intuitivas e facilmente identificáveis aos seus usuários. Os itens que receberam notas mais baixas dos usuários participantes deste estudo foi aprender a operar $(5,0)$ e a executar tarefas $(5,32)$ especialmente as mais complexas do sistema. Sendo assim, pode-se relacionar a menor satisfação do usuário ao aprender a operar e executar tarefas com os problemas de usabilidade verificados nos testes de usabilidade aplicados com os participantes deste estudo, considerando a dificuldade destes em realizar tarefas que geralmente são menos utilizadas. 


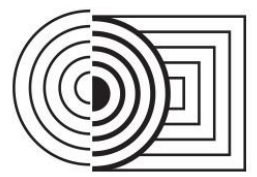

\section{$17^{\circ}$ ERGODESIGN \& USIHC 2019 \\ PUC-Rio, 11 a 13 de dezembro Rio de Janeiro, RJ, Brasil}

Além disto, o resultado deste estudo indica que a disposição dos elementos da tela $(6,05)$, principalmente relacionados às configurações do serviço de email, não proporciona um adequado grau de satisfação na interação do usuário com o sistema, conforme observado também por meio do Teste de Usabilidade e Avaliação Cooperativa.

Apesar da média de satisfação com a terminologia empregada no Gmail ser de 7,21, durante a avaliação cooperativa observou-se um problema no termo "marcador" utilizado pela ferramenta de correio eletrônico na organização dos emails recebidos na caixa de entrada em pastas. Este termo não foi intuitivamente associado com o recurso de organização de mensagens recebidas na caixa de entrada, por assunto em comum, em pastas.

O tempo de realização das tarefas realizadas, tanto nos testes de usabilidade quanto na avaliação cooperativa, foram relativamente semelhantes quando comparadas com cada tarefa específica. Sendo a tarefa "criar um evento e enviar convites" aquela que demandou mais tempo e a tarefa "configurar o tempo de cancelamento de envio de um email" o menor tempo. Isso justifica-se, pois a tarefa que precisou de mais tempo tinha uma série de ações para sua execução. Importante salientar, também, que para o usuário realizar a última tarefa "configurar o tempo de cancelamento de envio de um email" já havia explorado algumas opções, sendo assim, houve um tempo de contato prévio com a interface do Gmail. Foi possível observar que embora haja a opção ajuda, apenas um usuário tentou pesquisála. Isso demonstra o desinteresse, desinformação ou a percepção que a opção "ajuda" pouco contribuiria para a realização das tarefas. Cabe, portanto, uma análise mais detalhada e por consequência ajustes para que seja mais atraente e eficiente aos usuários do Gmail.

\section{CONSIDERAÇÕES FINAIS}

A usabilidade tem cada vez mais relevância e importância, sobretudo na perspectiva dos usuários, pois permite a utilização dos produtos e interfaces de forma fácil, eficiente e agradável ao contribuir para realização das tarefas de forma segura e eficiente, evitando situações indesejáveis, bem como, atingir as necessidades e expectativas dos usuários. Sabe-se que o correio eletrônico é uma ferramenta muito usada como meio de comunicação seja pessoal ou profissional mas é preciso considerar que seu uso correto e a utilização das funcionalidades disponíveis são essenciais para realizar e facilitar as tarefas dos usuários.

Podemos verificar que a satisfação geral dos usuários participantes da pesquisa com o sistema Gmail obteve uma média de 7,79. A análise das tarefas e da interação com o sistema demonstrou que apesar de quase a totalidade dos usuários participantes conseguirem executar as tarefas analisadas, algumas delas apresentaram certo grau de dificuldade e insatisfação.

Conforme a comparação com os dados do questionário de background e as tarefas executadas, os usuários com mais experiência no uso de correios eletrônicos conseguiram realizar as tarefas com mais facilidade e no menor tempo. Isso independente se o Gmail era seu email prioritário e se já tinha experiência com a tarefa solicitada. Demonstrando assim a 


\section{$17^{\circ}$ ERGODESIGN} \& USIHC 2019

PUC-Rio, 11 a 13 de dezembro

Rio de Janeiro, RJ, Brasil $17^{\circ}$ Ergodesign - Congresso Internacional de Ergonomia e Usabilidade de Interfaces Humano Tecnológica: Produto, Informações Ambientes Construídos e Transporte

$17^{\circ}$ USIHC - Congresso Internacional de Ergonomia e Usabilidade

de Interfaces Humano Computador

necessidade de reforçar o componente intuitivo e com uma interface acessível a todos, independente do grau de experiência.

Por fim, podemos concluir que os problemas encontrados nas tarefas específicas podem ser solucionados adequando o Gmail aos princípios de usabilidade para atender as

necessidades de interação com os usuários.

\section{REFERÊNCIAS BIBLIOGRÁFICAS}

CALORE, M. Gmail. Wired, v. 21, n. 5, p. 82-82, 2013.

CYBIS, W. Engenharia de Usabilidade: Uma abordagem Ergonômica. Florianópolis: UFSC, 2003. $138 \mathrm{p}$.

LINS, E.F.B. A evolução da Internet: uma perspectiva histórica.Brasília:cadernos ASLEGIS, p.11-45, 2013.

MONK, A.; WRITGHT, P.; HABER, J.; DAVENPORT L. Imporving your human-computer interface: a practical technique. London: Prentice-Hall, 1993.

RUBIN, J.; CHISNELL, D. Handbook of Usability Testing: How to Plan, Design, and Conduct Effective Tests. Indianapolis: Wiley Publishing, 2008.

SANTA ROSA, J.G.; PEREIRA JUNIOR, A.; LAMEIRA, A. P. Neurodesign: o cérebro e a máquina. Rio de Janeiro: Rio Books, 2016.

SCHEN, J.; BRDICZA, O.; RUAN, Y. A comparision study of user behavior on Facebook and Gmail. Computers in Human Behavior, v. 29, p. 2650-2655, 2013.

SHNEIDERMAN, B. Designing the user interface: estrategies for effective humancomputer interaction. 4.ed. Pearson Education, Inc. 2005

NIELSEN, J. Usability Engineering. San Francisco: Morgan Kauffman, 1994.

\section{AGRADECIMENTOS}

Agradecemos a todos os participantes desta pesquisa por dedicarem parte de seu tempo a este estudo. 